\title{
Telarquia y estado nutricional: Estudio epidemiológico en dos grupos étnicos
}

\author{
Patricia Bustos' ${ }^{1}$ H ugo Amigo ${ }^{1 a}$, Santiago Muzzo², \\ Ximena 0 ssa 3 .
}

\section{Thelarche and nutritional status: An epidemiological study of two ethnic groups}

Background: Improvements in environmental conditions may result in an earlier onset of thelarche. However, its onset is not homogeneous among different population groups. Aim: To assess the relationship between nutritional status, ethnicity and age of thelarche. Material and methods: Cross-sectional study of girls in second through sixth grade, attending 165 schools located in Chile's Araucania region. Of these, 231 girls who presented thelarche (breast button) were selected. The girls' surnames were used to identify their ethncity: indigenous had three or four last names of Mapuche indigenous origin ( $\mathrm{n}=113$ ), while non-indigenous were those who only had Chilean-Spanish last names $(\mathrm{n}=118)$. Weight, height, waist circumference and skinfold thicknesses were measured and socio-economic background information was collected through a home interview. Results: The median age of thelarche was 10 years and 4 months, regardless of ethnic group. Multivariant models showed that an increase of one $\mathrm{z}$ score unit of body mass index (BMI) decreased the median age of thelarche by 5.6 months (CI: - 7.24 to -3.90), controlling for the effect of different covariables. A decrease in one z score of height retards the median age of thelarche by 5.5 months (CI: 4.02 to 6.98). Ethnicity did not influence the age of thelarche. Conclusions: The age of thelarche found by us is similar to that reported in international studies, it comes earlier as weight increases, is delayed as height decreases and is not related to ethnicity (Rev Méd Chile 2009; 137: 1301-8).

(Key words: Ethnic groups; Nutritional status; Sexual maturation)

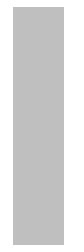

\footnotetext{
Recibido el 23 de enero, 2009. Aceptado el 7 de septiembre, 2009.

Trabajo financiado por Fondecyt, proyecto $\# 1060884$.

${ }^{1}$ Departamento de Nutrición, Facultad de Medicina, Universidad de Chile. Santiago de Chile. ${ }^{2}$ Instituto de Nutrición y Tecnología de los Alimentos, Universidad de Chile. ${ }^{3}$ Departamento de Obstetricia y Ginecología, Facultad de Medicina, Universidad de La Frontera. Temuco, Chile.

${ }^{a}$ Doctor en Salud Pública
}

Correspondencia a: Patricia Bustos. Departamento de Nutrición, Facultad de Medicina, Universidad de Chile. Independencia 1027, Santiago, Chile. Teléfono: 9786213. Fax: 7355581. E mail: pbustos@med.uchile.cl 
E n las niñas, la telarquia ocurre por la activación del eje hipotálamo-hipófisis-gonadal, marcando en la mayoría de los casos, el inicio del desarrollo puberal. En este proceso así como en la aparición del resto de los caracteres sexuales secundarios, participan diversos factores, tanto genéticos como ambientales ${ }^{1}$. Entre estos últimos, influye el estado nutricional, ya que se ha señalado que el "estirón puberal" se inicia con un peso crítico de $30,6 \mathrm{~kg}$, independiente de la edad o la talla ${ }^{2}$.

Diversos estudios han señalado que últimamente habría un adelanto secular del desarrollo puberal producto de las mejores condiciones de vida de las poblaciones que incluyen -entre otros factores- una alimentación más variada y suficiente, un mejor acceso a la atención de salud y posiblemente disruptores ambientales de tipo no hormonal ${ }^{3,4}$. Sin embargo, también se ha postulado que debido a la tendencia al aumento del exceso de peso que se ha observado en las últimas décadas, se podría esperar un adelanto en la presentación de los "eventos" puberales en años más recientes 5 .

Es conocido el aumento de la obesidad que han experimentado los escolares en el país ${ }^{6}$ y las consecuencias de esta patología en edades posteriores, al asociarse a diversas enfermedades crónicas no transmisibles ${ }^{7,8}$. En un estudio realizado en Chile sobre la edad en que se presenta la telarquia, se ha indicado que ocurriría antes de los 9 años?. No obstante, este resultado genera controversia, ya que este adelanto no se ha acompañado de una anticipación de la menarquia. Lo anterior indica la necesidad de más estudios para precisar esta información y para analizar la asociación entre exceso de peso y edad de telarquia.

Se ha señalado que el crecimiento durante el desarrollo de la pubertad es un determinante de la estatura final, por lo que el adelanto de la telarquia o menarquia podría contribuir a determinar una menor talla final en las niñas. En estudios anteriores se ha observado que los niños indígenas como no indígenas al nacer tienen una talla similar y crecen sin diferencias significativas hasta los 6 años si tienen condiciones medioambientales adecuadas ${ }^{10}$. Sin embargo, se ha observado que la estatura de los padres de los niños de procedencia indígena es menor que la mediana de estatura a los 18 años de la referencia internacional y más baja que la de sus pares no indígenas, especialmente las madres de estos niños ${ }^{11}$. Además, se ha informado que más de la mitad de los niños indígenas que ingresan a la escuela tienen sobrepeso y obesidad ${ }^{12}$. Estos hallazgos pudieran estar relacionados con diferencias en la edad en que se producen los eventos puberales en niñas que tienen diferente procedencia étnica.

El objetivo de este estudio fue determinar la edad de telarquia y el efecto que tienen el origen étnico y el estado nutricional en la edad de telarquia, en niñas que asistían a escuelas de la región de La Araucanía. Este trabajo se enmarca en el proyecto "Características del crecimiento durante la adolescencia: un estudio interétnico".

\section{MATERIAL Y MÉTODO}

Esta es la primera parte de un estudio de seguimiento de dos cohortes entre eventos puberales: una de niñas indígenas y otra de no indígenas. La muestra estuvo constituida por la totalidad de las niñas que asistían a 165 escuelas de 20 comunas de la región de La Araucanía (entre $2^{\circ}$ y $6^{\circ}$ año básico, $n=1.461$ ) quienes fueron examinadas para identificar la presencia del botón mamario mediante palpación, teniendo presentes los grados de desarrollo mamario propuestos por Tanner, denominándose "mama con desarrollo grado II" a la presencia de un aumento de volumen de la glándula mamaria de 1 a $2 \mathrm{~cm}$, de consistencia mayor que el tejido adyacente, generalmente dolorosa al tacto, a veces con desarrollo asimétrico, la que se acompañó de la ubicación por parte de la niña en fotos de los estadios de desarrollo mamario de Tanner ${ }^{13}$

De las niñas examinadas, sólo 231 (16\%), quedaron clasificadas en estadio II de Tanner: 113 mapuche y 118 no mapuche. A todas se les realizó una evaluación antropométrica determinando su peso corporal mediante una balanza automática, con precisión de 100 g y la estatura en un antropómetro con una precisión de $1 \mathrm{~mm}$. Ambas mediciones se hicieron con las niñas descalzas y con un mínimo de ropa, en instrumentos calibrados periódicamente. Con estos 
datos se calculó el índice de masa corporal (IMC) (peso/talla ${ }^{2}$. Se consignaron además el perímetro de brazo y de cintura (que se midieron con una huincha no extensible en la parte media del brazo el primero y en la distancia media entre el borde costal y el hueso de la cadera el segundo) y los pliegues cutáneos bicipital, tricipital, subescapular y suprailíaco, con un caliper (marca Harpenden). El examen físico y las mediciones las efectuaron 3 matronas previamente entrenadas por un endocrinólogo (uno de los autores de este trabajo), quienes evaluaron en forma independiente, a una proporción de las niñas (según distribución por comunas), las profesionales fueron estandarizadas en la realización de estos procedimientos y permanentemente supervisadas. La estandarización de las mediciones se realizó siguiendo técnicas aceptadas internacionalmente ${ }^{14}$.

En este estudio, se contó con información adicional al examen de la glándula mamaria que permitió precisar con mayor certeza que las niñas incorporadas al estudio estaban en etapa de telarquia. Estos antecedentes fueron: radiografía de edad ósea, la que se comparó con la edad cronológica, autoevaluación de las niñas, mediante fotos, de los grados de desarrollo puberal, crecimiento en estatura de un control a otro (ya que esta comunicación es la primera parte de un estudio de seguimiento cada tres o cuatro meses de las niñas hasta la menarquia) y tiempo transcurrido entre telarquia y menarquia. Con todos estos antecedentes dos pediatras establecieron que la escolar se encontraba en etapa de telarquia.

Para la identificación de la procedencia étnica, se consideraron los dos apellidos de cada progenitor formándose un grupo de niñas indígenas que tenían 3 ó 4 apellidos de origen mapuche y un grupo de no indígenas, con 4 apellidos de origen chileno-español. No se incluyeron niñas con apellidos extranjeros o hijas naturales en que se desconocía el origen del apellido de alguno de los progenitores.

Se construyeron bases de datos mediante digitación doble y el análisis de la información se realizó en el programa SPSS versión 15. Las medidas antropométricas fueron analizadas en forma continua. No se trabajó con las variables categorizadas en obesidad o talla baja ${ }^{15}$ por la escasa prevalencia de esta condición en la muestra estudiada.

Inicialmente se analizaron desbalances entre variables, luego se compararon medidas de tendencia central y se realizaron pruebas de asociación para posteriormente construir modelos multivariados, mediante regresión lineal, para ver el efecto de ser de procedencia indígena y del incremento del peso o la disminución de la talla en la edad de la telarquia, previa verificación de efectos de interacción y confusión entre las variables.

Este proyecto contó con la aprobación del Comité de Ética de la Facultad de Medicina de la Universidad de Chile. A las niñas examinadas se les explicó en qué consistía el estudio, se les solicitó asentimiento personal para su participación y la firma de un consentimiento informado de los padres.

\section{Resultados}

La telarquia se presentó a los 10 años y 5 meses en las niñas de procedencia mapuche (edad mínima: 99,1 y máxima: 165,6 meses), dos meses después que en las no indígenas (10 años 3 meses, edad mínima: 96,8 y máxima: 157,5 meses) pero esta diferencia no alcanzó significación estadística. La mediana de escolaridad de las madres y padres de las niñas mapuches fue inferior en 3 años a las de los progenitores de las niñas no indígenas. Aproximadamente la mitad de las niñas estudiadas vivían en el área rural y esta proporción llegó casi a $80 \%$ en las de procedencia mapuche ( $\mathrm{p}<0,01)$.

Las medianas de peso y talla al momento del examen fueron $34,9 \mathrm{~kg}$ y $137,6 \mathrm{~cm}$, sin diferencias significativas por etnia. Estos valores expresados en medidas estandarizadas corresponden a cifras de IMC sobre la mediana $(0,38)$ y en talla, bajo la mediana (-0,34). El perímetro del brazo, circunferencia de cintura e IMC fueron significativamente mayores en las niñas de procedencia mapuche. La obesidad y talla baja se presentaron con escasa magnitud $(5,0 \%$ y $2,1 \%$ respectivamente), sin diferencias por grupo (Tabla 1).

En el análisis univariado, se pudo constatar que el tener ascendencia mapuche no modifica la edad de la telarquia como tampoco lo hacen 
Tabla 1. Características generales y antropométricas de escolares de sexo femenino de la región de La Araucanía, al momento de la telarquia

\begin{tabular}{|c|c|c|c|c|}
\hline Variable & $\begin{array}{l}\text { Total } \\
231\end{array}$ & $\begin{array}{l}\text { Indígenas } \\
\text { ( } \mathrm{n}=113 \text { ) }\end{array}$ & $\begin{array}{l}\text { No Indígenas } \\
(\mathrm{n}=118)\end{array}$ & Significancia \\
\hline Edad (meses)* & $\begin{array}{c}124,0 \\
(115,3 \text { a } 130,0)\end{array}$ & $\begin{array}{c}124,7 \\
(116,8 \text { a } 131,3)\end{array}$ & $\begin{array}{c}123,2 \\
(115,3 \text { a } 129,5)\end{array}$ & $0,364^{* *}$ \\
\hline $\begin{array}{l}\text { Escolaridad de las } \\
\text { madres (años)* }\end{array}$ & $\begin{array}{c}8,0 \\
(6 \text { a } 11)\end{array}$ & $\begin{array}{c}6,0 \\
(5 \text { a } 8)\end{array}$ & $\begin{array}{c}9,0 \\
(6 \text { a } 12)\end{array}$ & $0,0001^{* * *}$ \\
\hline $\begin{array}{l}\text { Escolaridad de los } \\
\text { padres (años)* }\end{array}$ & $\begin{array}{c}8,0 \\
(6 \text { a } 12)\end{array}$ & $\begin{array}{c}7,0 \\
(4 \mathrm{a} 8)\end{array}$ & $\begin{array}{c}11,0 \\
(8 \text { a } 12)\end{array}$ & $0,0001^{* * *}$ \\
\hline Vive en área rural (\%) & 55,0 & 78,6 & 32,2 & $0,0001+$ \\
\hline Peso $(\mathrm{kg}) *$ & $\begin{array}{c}34,9 \\
(31,7 \text { a } 37,8)\end{array}$ & $\begin{array}{c}35,7 \\
(32,5 \text { a } 38,9)\end{array}$ & $\begin{array}{c}34,3 \\
(30,7 \text { a } 37,3)\end{array}$ & $0,364^{* *}$ \\
\hline Talla $(\mathrm{cm})^{*}$ & $\begin{array}{c}137,6 \\
(133,2 \text { a } 141,4)\end{array}$ & $\begin{array}{c}137,8 \\
(132,8 \text { a } 141,0)\end{array}$ & $\begin{array}{c}137,4 \\
(133,3 \text { a } 142,3)\end{array}$ & $0,697^{* *}$ \\
\hline Talla (puntajes z) & $\begin{array}{c}-0,34 \\
(-0,90 \text { a } 0,25)\end{array}$ & $\begin{array}{c}-0,43 \\
(-0,99 \text { a } 0,02)\end{array}$ & $\begin{array}{c}-0,24 \\
(-0,83 \text { a } 0.34)\end{array}$ & $0,154^{* * *}$ \\
\hline Perímetro del brazo $(\mathrm{cm})^{*}$ & $\begin{array}{c}21,2 \\
(20,0 \text { a } 22,8)\end{array}$ & $\begin{array}{c}21,7 \\
(20,4 \text { a } 22,8)\end{array}$ & $\begin{array}{c}20,8 \\
(19,7 \text { a } 22,3)\end{array}$ & $0,004^{* *}$ \\
\hline $\begin{array}{l}\text { Circunferencia de } \\
\text { cintura }(\mathrm{cm})^{*}\end{array}$ & $\begin{array}{c}64,0 \\
(60,5 \text { a } 68,8)\end{array}$ & $\begin{array}{c}65,2 \\
(62,0 \text { a } 70,0)\end{array}$ & $\begin{array}{c}62,3 \\
(58,6 \text { a } 65,5)\end{array}$ & $0,001^{* *}$ \\
\hline $\begin{array}{l}\text { Suma de } \\
\text { pliegues }(\mathrm{mm})^{*}\end{array}$ & $\begin{array}{c}37,4 \\
(30,6 \text { a } 46,0)\end{array}$ & $\begin{array}{c}38,3 \\
(29,6 \text { a } 44,9)\end{array}$ & $\begin{array}{c}35,1 \\
(33,2 \text { a } 47,8)\end{array}$ & $0,033^{* *}$ \\
\hline $\operatorname{IMC}\left(\mathrm{kg} / \mathrm{m}^{2}\right)^{*}$ & $\begin{array}{c}18,2 \\
(16,9 \text { a } 19,9)\end{array}$ & $\begin{array}{c}19,0 \\
(17,5 \text { a } 20,0)\end{array}$ & $\begin{array}{c}17,7 \\
(16,6 \text { a } 19,3)\end{array}$ & $0,001^{* *}$ \\
\hline IMC (puntajes z) * & $\begin{array}{c}0,38 \\
(-0,05 \text { a } 0,96)\end{array}$ & $\begin{array}{c}0,59 \\
(0,13 \text { a } 1,26)\end{array}$ & $\begin{array}{c}0,21 \\
(-0,16 \text { a } 0,84)\end{array}$ & $0,001^{* *}$ \\
\hline Obesidad (\%) & 5,0 & 5,1 & 5,0 & 0,592 \\
\hline
\end{tabular}

*Los valores expresan la mediana y el espacio intercuartil. **Significancia medida por el test de la mediana.

${ }^{+}$Significancia medida por la prueba de chi cuadrado.

el vivir en el área rural ni las mediciones de adiposidad (circunferencia de cintura, sumatoria de pliegues). El aumento de un punto en el IMC (que corresponde a esta edad, aproximadamente $2 \mathrm{~kg}$ ) sí ejerce un efecto, adelantando en 5 meses la edad de telarquia $(\mathrm{p}<0,01)$. En la misma dirección actúa la escolaridad de las madres al observarse que al aumentar 1 año de estudio, la edad de la telarquia se adelanta en aproximadamente medio mes. La menor talla actúa en forma inversa al aumento del IMC, es decir por cada puntaje $\mathrm{z}$ que disminuye, se atrasa la edad de telarquia en casi 5 meses (Tabla 2).
En el modelo multivariado, en que se ajustó por etnia y dos variables socioeconómicas (escolaridad de la madre y ruralidad), se pudo comprobar que el tener un puntaje z más de IMC adelanta en cinco y medio meses la edad de la telarquia (Tabla 3). Por su parte, después del ajuste, por las mismas variables utilizadas para el análisis anterior, se pudo constatar que la disminución de 1 puntaje $\mathrm{z}$ de talla (que corresponde para esta edad a aproximadamente $7 \mathrm{~cm}$ ), retrasa en cinco y medio meses la edad de la telarquia (Tabla 4). Si se repite este último análisis controlando además el peso $(\mathrm{kg})$, se observa que el coeficiente B cambia a 
Tabla 2. Asociación entre factores socioeconómicos, nutricionales y procedencia étnica en la edad de la telarquia (análisis univariado)

\begin{tabular}{|lcrrr|}
\hline Variables & $\begin{array}{c}\text { Variación de la edad } \\
\text { de la telarquia (meses) } \\
\text { Coeficiente B }\end{array}$ & $\begin{array}{c}\text { Intervalo } \\
\text { de confianza } \\
\text { inferior }\end{array}$ & superior & \\
\hline Ser indígena & 1,507 & $-1,393$ & 4,406 & 0,307 \\
Aumento de la escolaridad materna & $-0,426$ & $-0,840$ & $-0,011$ & 0,044 \\
Vivir en área rural & 2,493 & $-0,410$ & 5,395 & 0,092 \\
Tener un cm más de cintura & 0,004 & $-0,006$ & 0,014 & 0,390 \\
Aumento de la suma de pliegues & $-0,099$ & $-0,221$ & 0,023 & 0,110 \\
Tener un puntaje z más de IMC & $-5,142$ & $-6,795$ & $-3,490$ & 0,0001 \\
Tener un puntaje z menos de talla & 5,398 & 3,931 & 6,865 & 0,0001 \\
\hline
\end{tabular}

Tabla 3. Asociación entre el aumento del IMC y edad de la telarquia (análisis multivariado)

\begin{tabular}{|lcccc|}
\hline Variables & $\begin{array}{c}\text { Variación de la edad } \\
\text { de telarquia, en meses } \\
\text { (Coeficiente B) }\end{array}$ & \multicolumn{2}{c}{$\begin{array}{c}\text { Intervalo } \\
\text { de confianza } \\
\text { inferior }\end{array}$} & superior \\
& $-5,573$ & $-7,243$ & $-3,903$ & \\
\hline Aumento de un puntaje z de IMC & 1,691 & $-1,479$ & 4,860 & 0,0001 \\
Ser indígena & $-0,327$ & $-0,747$ & $-0,093$ & 0,127 \\
Aumento de la escolaridad materna & 1,832 & $-1,284$ & 4,947 & 0,248 \\
Vivir en área rural & & & & \\
\hline
\end{tabular}

Tabla 4. Asociación entre disminución de la estatura y edad de la telarquia (análisis multivariado)

\begin{tabular}{|lcccc|}
\hline Variables & $\begin{array}{c}\text { Variación de la edad } \\
\text { de telarquia, en meses } \\
\text { C oeficiente B }\end{array}$ & $\begin{array}{c}\text { Intervalo } \\
\text { de confianza } \\
\text { inferior }\end{array}$ & superior & \\
\hline Disminución de un puntaje z de talla & 5,501 & 4,021 & 6,980 & 0,0001 \\
Ser indígena & $-1,153$ & $-4,233$ & 1,928 & 0,462 \\
Aumento de la escolaridad materna & $-0,313$ & $-0,725$ & 0,100 & 0,137 \\
Vivir en el área rural & 2,098 & $-0,961$ & 5,157 & 0,178 \\
\hline
\end{tabular}

8,592 (IC: 7,134-10,051), es decir la edad de telarquia se atrasa aún más (datos no mostrados en tablas). No se encontró interacción entre las variables.
DisCUSIÓN

En este estudio se comprobó que la telarquia en la región de La Araucanía se presenta a los 10 años y 
4 meses, se adelanta con el exceso de peso, se atrasa con el déficit de talla y que este evento puberal no está influenciado por la etnia mapuche, la ruralidad, ni otros factores socioeconómicos.

La edad de telarquia encontrada en este estudio no concuerda con la reportada por Codner y cols quienes detectaron en niñas de algunas escuelas de la ciudad de Santiago una edad promedio de su estadio 2 de mamas según Tanner de 8,88 años 9 , pero sí está más de acuerdo con lo descrito en otros artículos internacionales y nacionales en que la edad de la telarquia es después de los 10 años ${ }^{16-18}$.

El adelanto secular de los eventos puberales, se debería a mejorías socioeconómicas que han experimentado los países en décadas más recientes, lo que se ha descrito principalmente para la menarquia, con edades variables según el país analizado ${ }^{19,20}$. Aunque también se ha indicado para la telarquia ${ }^{21}$, esta tendencia no es similar para todos los grupos poblacionales ya que en Estados Unidos de Norteamérica, entre 1982 y 1994, se observó un adelanto especialmente en niñas afroamericanas ${ }^{22}$. Cabe destacar que en el estudio de Codner en las escolares de Santiago9, el adelanto de la telarquia no se acompañó por la anticipación de la menarquia, lo que implicaría una mayor duración del desarrollo puberal.

También, se ha descrito que telarquia y menarquia se anticiparía en niñas que tienen mayor IMC o con exceso de peso ${ }^{23-27}$. Este efecto sí fue observado en nuestro estudio al comprobar un adelanto cercano a los 6 meses en la edad de aparición del botón mamario en las niñas que tenían un aumento del IMC. El efecto de la obesidad no pudo ser explorado, por la limitada prevalencia de ella en la muestra. Este hallazgo es curioso, ya que la prevalencia de obesidad que se reporta para los escolares al ingreso de la escuela es muy superior ${ }^{6}$, lo que plantea la necesidad de revisar la referencia que se utiliza en el país en escolares prepúberes y púberes, pero, también es necesario decir que esto no modifica los resultados de este estudio.

Hay estudios que han señalado que el adelanto del inicio del desarrollo puberal se debería a la hiperinsulinemia y resistencia a la insulina ${ }^{28}$ que se ve en la actualidad con mayor frecuencia asociada a obesidad y que se ha atribuido al cambio en los estilos de vida de la población, asociación que requiere mayor estudio. Otros, han comunicado que el aumento de la leptina detectado en sujetos obesos favorecería el inicio temprano del desarrollo puberal, dado su función inductora de la pubertad ${ }^{29}$.

La asociación de menor talla con retraso en la telarquia está en concordancia con el atraso de edad ósea y por tanto de los eventos puberales observados en niñas desnutridas ${ }^{30}$. En relación a estos resultados, se hace necesario el estudio de la velocidad de crecimiento entre eventos puberales, en niñas con exceso de peso y con déficit de talla, dado que pueden terminar con estaturas muy distintas influenciadas no sólo por el momento en que ocurren los eventos puberales sino por el estado nutricional con el cual se inicia esta etapa del desarrollo.

Una de las restricciones de los estudios sobre la edad de telarquia es que muchos de ellos la determinan por inspección, o bien por la autoevaluación al observar una foto con los diferentes estadios del desarrollo mamario de Tanner, lo que puede producir una imprecisión en su determinación. Es necesario mencionar las dificultades para el establecimiento del grado II de Tanner en niñas con exceso de peso, ya que la palpación de la glándula se dificulta, pero destaca la importancia de la palpación de la glándula al examen físico para precisar su presencia y evitar confundir una lipomastia con telarquia. Otra limitación es que la telarquia no se presenta sólo en un momento en el tiempo sino en un intervalo de tiempo que puede durar 6 meses o más, y que por lo tanto existen diferentes tamaños y características del botón mamario en el estadio II de desarrollo. Sin embargo, pese a las dificultades mencionadas, y como se señaló en la metodología, en este estudio, se contó con información adicional al examen de la glándula mamaria que permitió precisar con mayor certeza que las niñas incorporadas al estudio estaban en etapa de telarquia.

Entre las fortalezas de nuestro estudio se puede mencionar que la telarquia se identificó por palpación de la glándula, la que fue efectuada por profesionales entrenadas y permanentemente supervisadas. Además, que se trabajó con 
gran acuciosidad en la búsqueda de la muestra y rigurosidad en la definición de etnia y que se ha efectuado en la región en que mayoritariamente viven las personas pertenecientes a la etnia mapuche en nuestro país. Este estudio es el único que indica la edad de telarquia en población indígena lo que puede entregar valiosos antecedentes para conocer las características del

\section{REFERENCIAS}

1. García H, Ugarte F. Trastornos de la pubertad. En: Rizzardini M, Ed. Pediatría, Capítulo 19. Editorial Mediterráneo 1999.

2. Johnston FE, Malina RM, Galbraith MA, Frisch RE, Revelle R, CoOK S. Height, weight and age at menarche and the "critical weight" hypothesis. Science 1971; 174: 1148-9.

3. Ong KK, Ahmed ML, Dunger DB. Lessons from large population studies on timing and tempo of puberty (secular trends and relation to body size): the European trend. Mol Cell Endocrinol 2006; 254-255: 8-12.

4. Graham MJ, Larsen U, Xu X. Secular trend in age at menarche in China: a case study of two rural counties in Anhui Province. J Biosoc Sci 1999; 31: 257-67.

5. Kaplowitz P. Pubertal development in girls: secular trends. Curr Opin Obstet Gynecol 2006; 18: 487-91.

6. Vio F, Albala C, Kain J. Nutrition transition in Chile revisited: mid-term evaluation of obesity goals for the period 2000-2010. Public Health Nutr 2008; 11 : 405-12.

7. Battista M, Murray RD, Daniels SR. Use of the metabolic syndrome in pediatrics: a blessing and a curse. Semin Pediatr Surg 2009; 18: 136-43.

8. Iglesias P, Olivar J, Peñalver D, Díaz P, Vega B, Monereo S. [Effect of abdominal obesity on size of myocardial infarction]. Endocrinol Nutr 2009; 56: 4-8.

9. Codner E, Unanue N, Gaete X, Barrera A, MookKanomori D, Bazáez R et al. Cronología del desarrollo puberal en niñas escolares de Santiago: relación con nivel socioeconómico e índice de masa corporal. Rev Méd Chile 2004; 132: 801-8.

10. Bustos P, Amigo H, Muñoz S, Martorell R. Growth in indigenous and non indigenous Chilean schoolchildren from 3 poverty strata. Am J Public Health 2001; 91: $1645-9$. crecimiento y desarrollo en adolescentes de distintos grupos étnicos.

\section{Agradecimientos}

A las matronas Elba Contreras, Roxana Mella y Claudia Fernández quienes identificaron a las niñas que participaron en este estudio y realizaron las mediciones y entrevistas domiciliarias.

11. Amigo H, Erazo M, Bustos P. Estatura de padres e hijos chilenos de diferente etnia y vulnerabilidad social. Salud Pública Mex 2000; 42: 504-10.

12. Amigo H, Bustos P, Erazo M, Radrigán ME. Comparación del estado nutricional entre escolares indígenas y no indígenas. Rev Méd Chile 1999; 127: 903-10.

13. TAnner JM. Growth at adolescence, $2^{\text {nd }}$ edn. Oxford: Blackwell Scientific Publication, 1962.

14. De Onis M, Onyango AW, Van Den Broeck J, Chumlea WC, Martorell R. Measurement and standardization protocols for anthropometry used in the construction of a new international growth reference. Food Nutr Bull 2004; 25(Suppl): S27-36.

15. CDC/NCHS. CDC growth charts: Unites States 2000. Disponible en: http://www.cdc.gov/growthcharts. [Consultado el 2 de octubre de 2008].

16. Zukauskaite S, Lasiene D, Lasas L, Urbonaite B, HindMaRsh P. Onset of breast and pubic hair development in 1231 preadolescent Lithuanian schoolgirls. Arc Dis Child 2005; 90: 932-6.

17. Hosny LA, El-Ruby MO, ZaKi ME, Aglan MS, ZaKi MS, El Gammal MA et al. Assessment of pubertal development in Egyptian girls. J Pediatr Endocrinol Metab 2005; 18: 577-84.

18. Burrows R, Leiva L, Mauricci A, Zvaigghaft A, Muzzo S. Características de la pubertad en escolares de diferente nivel socioeconómico de la Región Metropolitana de Chile. Rev Chil Pediatr 1988; 59: 240-6.

19. Malina RM, Peña Reyes Me, Tan SK, Little BB. Secular change in age at menarche in rural Oaxaca, southern Mexico: 1968-2000. Ann Hum Biol 2004; 31: 634-46.

20. Silva HP, Padez C. Secular trends in age at menarche among Caboclo populations from Pará, Amazonia, Brazil: 1930-1980. Am J Hum Biol 2006; 18: 83-92.

21. Mahachoklertwattana P, Suthutvoravut U, CharoenkiatKul S, Chongviriyaphan N, Rojroongwasinkul N, ThaKKINSTIAN A ET AL. Earlier onset of pubertal maturation in Thai girls. J Med Assoc Thai 2002; 85 (Suppl 4): S1127-34. 
22. Sun SS, Schubert CM, Liang R, Roche AF, Kulin He, Lee PA ET AL. Is sexual maturity occurring earlier among US children? J Adolesc Health 2005; 37: 345-55.

23. Juul A, Teilmann G, Scheike T, Hertel NT, Holm K, Laursen EM et al. Pubertal development in Danish children: comparison of recent European and US data. Int J Androl 2006; 29: 247-55.

24. Ribeiro J, Santos P, Duarte J, Mota J. Association between overweight and early sexual maturation in Portuguese boys and girls. Ann Hum Biol 2006; 33: 55-63.

25. Himes JH, Obarzanek E, Baranowski T, Wilson DM, Rochon J, Mcclanahan BS. Early sexual maturation, body composition, and obesity in African-American girls. Obes Res 2004; 12 (Suppl): 64S-72S.

26. Kaplowitz PB, Slora EJ, Wasserman RC, Pedlow SE,
HeRman-Giddens ME. Earlier onset of puberty in girls: relation to increased body mass index and race. Pediatrics 2001; 108: 347-53.

27. Hernández Mi, Unanue N, Gaete X, Cassorla F, Codner E. Edad de la menarquia y su relación con el nivel socioeconómico e índice de masa corporal. Rev Méd Chile 2007; 135: 1429-36.

28. SLYPer AH. The pubertal timing controversy in the USA, and a review of possible causative factors for the advance in timing of onset of puberty. Clin Endocrinol (Oxf) 2006; 65: 1-8.

29. Kaplowitz PB. Link between body fat and the timing of puberty. Pediatrics 2008; 121 Suppl 3: S208-17.

30. Garnier D, Simondon KB, Benefice E. Longitudinal estimates of puberty timing in Senegalese adolescent girls. Am J Hum Biol 2005; 17: 718-30. 